\title{
Potential link between SARS-CoV-2 and Kawasaki disease: importance of dentists for the diagnosis
}

\author{
Hercílio MARTELLI-JÚNIOR(a) \\ Renato Assis MACHADO(b) (iD \\ Daniella Reis Barbosa MARTELLI(c) \\ Mauro Costa BARBOSA(c) \\ Paulo Rogério Ferreti BONAN(d) \\ Ricardo Della COLETTA(e) (iD \\ (a) Universidade de Montes Claros - Unimontes, \\ Dental School Health Sciences Postgraduate \\ Program, Montes Claros, MG, Brazil. \\ (b) Universidade de São Paulo - USP, School \\ of Dentistry, Hospital for Rehabilitation of \\ Craniofacial Anomalies, Bauru, SP, Brazil. \\ (c) Universidade de Montes Claros - \\ Unimontes, Dental School, Department of \\ Oral Diagnosis, Montes Claros, MG, Brazil. \\ (d) Universidade Federal da Paraíba - UFPB, \\ Health Science Centre, João Pessoa, \\ PB, Brazil. \\ (e) Universidade Estadual de Campnas - \\ Unicamp, School of Dentistry, Department \\ of Oral Diagnosis, Piracicaba, SP, Brazil.
}

Declaration of Interests: The authors certify that they have no commercial or associative interest that represents a conflict of interest in connection with the manuscript.

Corresponding Author:

Renato Assis Machado

E-mail: renatoassismachado@yahoo.com.br

https://doi.org/10.1590/1807-3107bor-2021.vol35.0047

Submitted: June 12, 2020

Accepted for publication: August 26, 2020

Last revision: February 2, 2020
Abstract: Kawasaki disease (KD) is a vasculitis with predilection for coronary arteries. Due to a lack of reliable confirmatory laboratory tests, the diagnosis of KD is based on a characteristic pattern of clinical findings that appear in a typical temporal sequence. The diagnostic criteria have been periodically modified and the American Heart Association has proposed the most recent guidelines for its diagnosis. However, patients may have incomplete or atypical forms of KD and diagnosis can often be difficult. Because oropharyngeal manifestations are a common and important feature for diagnosing KD and recent studies have hypothesized a possible association between KD and the severe acute respiratory syndrome coronavirus 2 (SARS-CoV-2), in this review we highlight the importance of dentists in the diagnosis of KD and its potential association with SARS-CoV-2.

Keywords: Mucocutaneous Lymph Node Syndrome; Coronavirus Infections; Dental Care.

\section{Introduction}

Kawasaki disease (KD, OMIM \#611775), a medium-sized vasculitis with coronary artery predilection, shows a variable incidence rate ranging from 4 to 300 per 100,000 children. ${ }^{1,2}$ It is more frequently diagnosed in boys and the diagnosis is based on clinical features, including recurrent fever, polymorphic rash, characteristic changes in the extremities (erythema of the hands and feet accompanied by desquamation of the fingers and toes), bilateral conjunctivitis, cervical lymphadenopathy, oropharyngeal changes, such as a 'strawberry' tongue (prominent lingual papillae), dry, erythematous or split-lips, and oropharyngeal mucosal erythema, ${ }^{3}$ because there is no gold standard laboratory test. ${ }^{4}$ KD was first identified in 1967 by Tomisaku Kawasaki in 50 Japanese children less than 5-years-old. Currently, nearly all racial groups are affected. ${ }^{5}$

Although the etiology of KD is unknown, some studies have described an association with viral respiratory infections. ${ }^{6,7}$ Turnier et al., ${ }^{6}$ for example, associated $28 \%$ of cases of KD with rhinovirus/enterovirus and $8.7 \%$ were attributed to parainfluenza. The association of KD and severe acute respiratory syndrome coronavirus 2 (SARS-CoV-2) was noticed after the first case of KD was diagnosed in a patient infected with the coronavirus disease 2019 (COVID-19) by Jones et al. ${ }^{7}$ Furthermore, a cluster 
of SARS-CoV-2-infected children presenting with KD-like symptoms has also been reported, as well as, multisystem inflammatory syndrome (MIS), KD shock syndrome, and toxic shock syndrome. . $^{8,10,11,12}$

This association has worried the scientific community, since the COVID-19 pandemic has reached more of 19.4 million cases and 721,000 deaths (https://who.sprinklr.com, accessed on Aug $9^{\text {th }}, 2020$ ). In Brazil, the first COVID-19 official case was reported on February 25, 2020, and currently the country has the most cases and deaths in Latin America $(3,012,412$ cases and 100,477 deaths as of August 8, 2020) (https://covid.saude.gov.br/), and these statistics are probably substantially underestimated. ${ }^{13}$

As orofacial manifestations are common and essential for diagnosing KD and a potential association with COVID-19 was reported, we highlight, in this critical review, the clinical and laboratory features of KD and its association with SARS-CoV-2.

\section{Discussion}

\section{Diagnosis criteria}

Two official guidelines for KD diagnosis have been developed by the Kawasaki Disease Research Committee (KDRC, 2002) ${ }^{14}$ and by the American Heart Association (AHA, 2017) ${ }^{15,16}$ (Tables 1 and 2). Those guidelines do not differ significantly from the original description by Kawasaki in 1967,7 and the main difference between the KDRC and AHA guidelines is that while fever for more than five days is an essential condition in the AHA's guidelines, it is not recognized in the KDRC's guidelines. However, in the AHA's guidelines, the importance of fever for more than five days is minimized if the patient shows more than four of the principal clinical signs of inflammation in mucocutaneous areas, including the skin (feet and hands) and mucous membranes, such as the oral cavity and eyes.

Although a specific diagnostic test does not exist, some findings may help to distinguish KD from other similar conditions. ${ }^{18}$ The disease usually shows three stages (average duration of 6 to 8 weeks) and different laboratory findings can occur. The first stage is the acute febrile period that lasts one to two weeks, with nonspecific results, but may help support the diagnosis in children who do not fulfil clinical criteria. There is an increase in the erythrocyte sedimentation rate, C-reactive protein, blood cell count (predominance of neutrophils), serum transaminase levels associated with hypoalbuminemia, thrombocytosis (this finding usually occurs more in the subacute phase), and anemia. The subacute period (around 25 days) is characterized by thrombocytosis in association with periungual desquamation of the fingers or toes. The last phase (convalescent phase, around four to eight weeks) shows a normal sedimentation rate and platelet count. ${ }^{19}$

Findings on high-resolution echocardiography also provide important information for KD diagnosis and treatment. ${ }^{20}$ Among the most common cardiac complications are coronary artery aneurysm, decreased myocardial contractility, congestive heart failure, arrhythmias, pancarditis, pericardial effusion, and myocardial ischemia. ${ }^{21}$ According to AHA guidelines, children with fever for more than five days and two or more clinical signs of mucocutaneous inflammation, plus coronary artery dilation and/or characteristic laboratory test results (Table 3 ) should be considered for KD diagnosis.

\section{Etiology and the relationship with SARS-CoV-2}

Although the etiology of KD is unknown, bacterial and viral infections are considered the most likely triggers of the disease. ${ }^{22}$ Among bacteria related to KD are Staphylococcus aureus, Streptococcus A, Yersinia pseudotuberculosis, Klebsiella pneumoniae, Pseudomonas aeruginosa, Chlamydiae pneumoniae, Mycoplasma pneumoniae and Rickettsial. The viruses frequently associated with KD are Parvovirus B19, Epstein Barr virus, Cytomegalovirus, Human herpesvirus 6, Rhinovirus/Enterovirus, Retrovirus, Rotavirus, Parainfluenza virus, New Haven coronavirus, Measles, Chickenpox, and Dengue virus, where $28 \%$ of KD cases are associated with Rhinovirus/Enterovirus. ${ }^{6,23}$

Epidemiological data from many countries show that children are affected by SARS-CoV-2 in far smaller proportions than adults, with only $2 \%$ of cases described in patients under the age of $20 .{ }^{24}$ Recently, in some countries, there has been an apparent cluster 
Table 1. Guidelines for the diagnosis of Kawasaki disease from Kawasaki Disease Research Committee (KDRC, 2002). ${ }^{14}$

The symptoms can be classified into two categories, principal symptoms and other significant symptoms or findings:

Principal symptoms

1 Fever that persists for 5 days or more (inclusive of cases in whom the fever has subsided before the fifth day in response to therapy);

2 Bilateral conjunctival congestion;

3 Changes of lips and oral cavity: reddening of the lips, strawberry tongue, and oral and pharyngeal mucosal diffuse injection;

4 Polymorphous exanthema;

5 Changes of peripheral extremities: (Initial stage) reddening of the palms and soles, indurative edema; (Convalescent stage) membranous fingertip desquamation;

6 Acute non-purulent cervical lymphadenopathy;

At least 5 items of the 6 should be satisfied for diagnosis of KD. However, patients with 4 items of the principal symptoms can be diagnosed with $\mathrm{KD}$ when coronary aneurysm or dilatation is recognized by 2-D echocardiography or coronary angiography.

Other significant symptoms or findings

The following symptoms and findings should be considered in the clinical evaluation of suspected patients:

1 Cardiovascular: auscultation (heart murmur, gallop rhythm, distant heart sounds), electrocardiogram changes (prolonged PR/QT intervals, abnormal $Q$ wave, low-voltage QRS complexes, ST-T changes, arrhythmias), chest X-ray findings (cardiomegaly), 2-D echo findings (pericardial effusion, coronary aneurysms), aneurysm of peripheral arteries other than coronary (eg, axillary), angina pectoris or myocardial infarction;

2 Gastrointestinal tract: diarrhea, vomiting, abdominal pain, hydrops of gall bladder, paralytic ileus, mild jaundice, slight increase of serum transaminase;

3 Blood: leukocytosis with shift to the left, thrombocytosis, increased erythrocyte sedimentation ratio, positive C-reactive peptide, hypoalbuminemia, increased $\alpha 2$-globulin, slight decrease in erythrocyte and hemoglobin levels;

4 Urine: proteinuria, increase of leukocytes in urine sediment;

5 Skin: redness and crust at the site of Bacille Calmette-Guèrin inoculation, small pustules, transverse furrows of the finger nails;

6 Respiratory: cough, rhinorrhea, abdominal shadow on chest $X$-ray;

7 Joint: pain, swelling; and

8 Neurological: cerebrospinal fluid pleocytosis, convulsion, unconsciousness, facial palsy, paralysis of the extremities.

Remarks

1 For item 5 under principal symptoms, the convalescent stage is considered important.

2 Non-purulent cervical lymphadenopathy is less frequently encountered (approximately 65\%) than other principal symptoms during the acute phase.

3 Male:Female ratio, 1.3-1.5: 1; patients under 5 years of age, $80-85 \%$; fatality rate, $0.1 \%$.

4 Recurrence rate, 2-3\%; proportion of siblings cases, $1-2 \%$.

5 Approximately $10 \%$ of the total cases do not fulfil 5 of the 6 principal symptoms, in which other diseases can be excluded and KD is suspected. In some of these patients, coronary artery aneurysms (including so-called coronary artery ectasia) have been confirmed.

of children presenting with symptoms of KD, which seems related to the COVID-19 pandemic. $712,25,26,27,28$ A French center, for example, diagnosed 17 cases in 11 days, in contrast to an average of two cases per month in 2018-2019. ${ }^{28}$ Similarly, Verdoni et al. ${ }^{11}$ have described KD-like symptoms in 10 SARS-CoV-2 positive Italian children in two months, in contrast with 19 cases diagnosed in the past five years. Of the children evaluated in the report by Verdoni et al., ${ }^{11}$ five showed typical KD features, including non-purulent conjunctivitis, polymorphic rash, mucosal changes and swollen extremities, and another five children showed fewer than three of the minimal clinical signs for KD diagnosis. There were also a high proportion of patients experiencing shock, with five out of ten children showing fluid resuscitation hypotension and two needing inotropic support. These differences raise the question of whether this cluster is KD triggered by SARS-CoV-2, or if it represents features related to multisystem inflammation.

While the SARS-CoV-2 could become endemic, like $\mathrm{HIV}$, the WHO, warn against any attempt to predict 
Table 2. Guidelines for the diagnosis of Kawasaki disease from American Heart Association (AHA, 2017). 15,16

$\mathrm{KD}$ is diagnosed in the presence of:

1 Fever persisting at least 5 days* and

2 At least 4 of the 5 principal clinical features:

i) Changes in extremities

Acute: Erythema of palms, soles; edema of hands, feet

Subacute: Periungual peeling of fingers and toes in weeks 2 and 3

ii) Polymorphous exanthema (diffuse maculopapular, urticarial, erythroderma, erythema-multiforme like, not vesicular or bullous)

iii) Bilateral bulbar conjunctival injection without exudate

iv) Changes in lips and oral cavity: erythema, lips cracking, strawberry tongue, diffuse injection of oral and pharyngeal mucosae

v) Cervical lymphadenopathy (> $1.5 \mathrm{~cm}$ diameter), usually unilateral

A careful history may reveal that $\geq 1$ principal clinical features were present during the illness but resolved by the time of presentation.

Patients who lack full clinical features of classic KD are often evaluated for incomplete KD. If coronary artery abnormalities are detected, the diagnosis of $\mathrm{KD}$ is considered confirmed in most cases.

Laboratory tests typically reveal normal or elevated white blood cell count with neutrophil predominance and elevated acute phase reactants such as $\mathrm{C}$-reactive protein and erythrocyte sedimentation rate during the acute phase. Low serum sodium and albumin levels, elevated serum liver enzymes, and sterile pyuria can be present. In the second week after fever onset, thrombocytosis is common.

Other clinical findings may include the following:

Cardiovascular: myocarditis, pericarditis, valvular regurgitation, shock, coronary artery abnormalities, aneurysms of medium-sized noncoronary arteries, peripheral gangrene, and aortic root enlargement.

Respiratory: Peribronchial and interstitial infiltrates on chest radiography and pulmonary nodules.

Musculoskeletal: Arthritis, arthralgia (pleocytosis of synovial fluid)

Gastrointestinal: Diarrhea, vomiting, abdominal pain, hepatitis, jaundice, gallbladder hydrops, and pancreatitis.

Nervous system: Extreme irritability, aseptic meningitis (pleocytosis of cerebrospinal fluid), facial nerve palsy, and sensorineural hearing loss.

Genitourinary: Urethritis/meatitis, hydrocele

Other: Desquamating rash in groin, retropharyngeal phlegmon, anterior uveitis by slit lamp examination, erythema and induration at bacillus Calmette-Guérin inoculation site.

The differential diagnosis includes other infectious and noninfectious conditions, including the following: Measles, other viral infections (eg, adenovirus, enterovirus), staphylococcal and streptococcal toxin-mediated diseases (eg, scarlet fever and toxic shock syndrome), drug hypersensitivity reactions, Stevens Johnson syndrome, systemic onset juvenile idiopathic arthritis, Rocky Mountain spotted fever or other rickettsial infections, and Leptospirosis.

*In the presence of $\geq 4$ principal clinical features, particularly when redness and swelling of the hands and feet are present, KD can be diagnosed even with 4 days of fever.

how long it may remain in circulation. This virus may become another endemic virus in our communities and may never go away (https://www.who.int/ emergencies/diseases/novel-coronavirus-2019). Due to the time interval of studies on the pandemic and the immunological mechanisms resulting from viral infection, many effects may be discovered.

\section{Principal clinical findings}

Usually, KD starts with a sudden, unremitting fever, of high intensity, lacking an antimicrobial response, and followed by inflammation of the mucosa. Although most children with KD have a fever which typically lasts longer than five days, however, this number is somewhat arbitrary. ${ }^{29}$ Thus, an experienced physician can diagnose KD on day three or four of the illness, in association with other clinical features. ${ }^{15}$ Such features include, bilateral exudative conjunctival injection with limbal sparing and the presence of erythema in the oral cavity and lips, with lip cracking found in $96 \%$ of patients. ${ }^{30}$

In addition, children with KD often have a non-specific erythema of the palms and soles and characteristic edema of the hands and feet, that usually subsides within a few days. Periungual skin peeling is a pathognomonic symptom of KD 
Table 3. Laboratory test and imaging results incorporated by American Heart Association for diagnosis of incomplete Kawasaki disease.

\begin{tabular}{lc}
\hline Laboratory Criteria & $\begin{array}{c}\text { Echocardiogram } \\
\text { Results }\end{array}$ \\
\hline C-reactive protein $>3.0 \mathrm{mg} / \mathrm{dL}$ & $\begin{array}{c}\text { Coronary z scores } \\
>2.5\end{array}$ \\
Erythrocyte sedimentation rate $>40 \mathrm{~mm} / \mathrm{h}$ & $\begin{array}{c}\text { Lack of tapering } \\
\text { Perivascular } \\
\text { brightness }\end{array}$ \\
Albumin $<3.0 \mathrm{~g} / \mathrm{dL}$ & $\begin{array}{c}\text { Decreased left } \\
\text { ventricular function }\end{array}$ \\
Anemia & $\begin{array}{c}\text { Mitral regurgitation } \\
\text { High rate alanine aminotransferase }\end{array}$ \\
$\begin{array}{l}\text { Platelets }>450 \times 10^{3} / \mu \mathrm{L} \text { after } 7 \text { days of } \\
\text { illness }\end{array}$ & \\
WBC $>15000 / \mu \mathrm{L}$ & \\
$>10$ WBCs $/$ high-power field on urinalysis & \\
\hline WBC: white blood cell count. &
\end{tabular}

usually occurring during the second or third week of illness. Although there is a report of periungual desquamation within 10 days, ${ }^{31}$ this is not very useful for an early diagnosis. ${ }^{32}$ Another non-specific lesion is the diffuse erythematous polymorphic rash that typically occurs in the first few days of illness. It can be seen in up to $96 \%$ of patients. However, it is often intermittent and may therefore be overlooked, particularly in children with dark skin. ${ }^{30}$ Unilateral cervical lymphadenopathy is a characteristic clinical sign of KD, but is seen in only $60 \%$ of patients and often mistaken for suppurative lymphadenitis. ${ }^{30}$

\section{Role of dentists on KD diagnosis}

Among mucocutaneous lesions found with KD, the most common occur in the orofacial region and can be found in most cases at time of diagnosis. ${ }^{16,33,34}$ The changes in the lips, including erythema, dryness, cracking, peeling, vertical cracking and bleeding, and changes in the mouth, such as strawberry tongue with erythema and prominent fungiform papillae, and diffuse oropharyngeal mucosa erythema, can occur both in the acute (0-9 days) and convalescence stages ( $>25$ days).$^{20}$ Oral mucositis, characterized by typical necrotizing microvasculitis with fibrinoid necrosis, is common in KD. ${ }^{34}$ Whittaker et al. ${ }^{12}$ have reported that $17(29 \%)$ of the patients with MIS presented mucus membrane changes and red, cracked lips, Cheung et al. $(2020)^{8}$ have found that
$53 \%$ of their patients presented lip-redness, and Verdoni et al. $(2020)^{11}$ have described that four of five patients $(80 \%)$ displayed associated changes of the lips or oral cavity, or both. These oral changes can be identified by dentists, and considering these together with the medical history, patients should be referred to the pediatrician for confirmation of a KD diagnosis. ${ }^{20}$

Early diagnosis of KD is essential, because the intravenous immunoglobulin (IVIG) administration within the first 10 days has shown to reduce the incidence of cardiac complications. ${ }^{21,33}$ Furthermore, a recent meta-analysis showed that changes in the oral mucosa is a clinical indicator of IVIG resistant in Asian patients and other alternative therapeutics are needed for those patients. ${ }^{35}$ Recurrence is reported at rates of $0.8 \%$ to $3 \%$, and oral manifestations can occur. ${ }^{36}$ Dry, cracked, red and swelling lips, and strawberry tongue are the most common signs of recurrence. ${ }^{36,37}$ Dentists should be aware that patients with KD can also have cardiac sequelae, requiring prophylactic antibiotics before invasive dental procedures.

Being a rare disease, some cases go unnoticed due to a lack of knowledge. Proper information assists in the timely diagnosis and reduces the mortality rate caused by the disease. Thus, the requirement of dentists to recognize KD is essential.

\section{Medical treatment and prognosis}

The treatment in the acute phase aims to reduce the inflammatory response in the coronary artery wall, preventing the consequences of this vasculitis, including thrombosis and aneurysm. ${ }^{22}$ A combination of a high-dose of IVIG (a single dose of $2 \mathrm{~g} / \mathrm{kg}$ ) and high-doses of acetylsalicylic acid (AAS; $80-100 \mathrm{mg} / \mathrm{kg} /$ day until the fourteenth day of illness and at least 48 to 72 hours after cessation of fever) which are administered within 10 days of illness or later if the patient has persistent fever, aneurysms or inflammation, is recommended. ${ }^{15}$ Subsequently, low-doses of AAS at 3 to $5 \mathrm{mg} / \mathrm{kg} /$ day is administrated for six to eight weeks, or throughout life if coronary complications occur. ${ }^{30}$ Although the anti-inflammatory role of AAS has been called into question and the IVIG's mechanism of action is unclear, ${ }^{15}$ this approach reduces the incidence 
of coronary artery defects from approximately $25 \%$ to less than $5 \%$, and giant aneurysms to $1 \%{ }^{22}$ However, it was reported that among $20-40 \%$ of patients with KD are resistant to IVIG therapy and have a significantly higher risk in developing coronary artery abnormalities than nonresistant patients. ${ }^{20,38}$ For IVIG-resistant KD patients, the protocol involves a second infusion of IVIG $(2 \mathrm{~g} /$ $\mathrm{kg}$ ) plus prednisolone $(2 \mathrm{mg} / \mathrm{kg}$ divided every $8 \mathrm{~h}$ until afebrile, then prednisone orally until C-reactive protein is normalized, then tapered over 2-3 weeks), or Infliximab (5 mg/kg given over $2 \mathrm{~h}$ ). Other alternative treatments are also used, such as cyclosporine, anakinra, cyclophosphamide, and plasma exchange. ${ }^{15}$ However, the roles of the repeated use of IVIG and other adjunctive therapies are uncertain, even though some evidence reports good results. ${ }^{30}$

KD patients in the subacute and convalescence phases with coronary aneurysms need to prevent thrombosis (platelet activation) and vessel stenosis. For this, low-dose aspirin (3-5 mg/kg/day) is the main therapy for children with small and medium aneurysms. ${ }^{20}$ Other antiplatelet agents are also used (clopidogrel, ticlopidine, dipyridamole) and their association with aspirin have been shown to be more effective in blocking platelet aggregation. ${ }^{20}$ Furthermore, the long-term risk of coronary disease results from intimate thickening and stenosis in regions adjacent to giant aneurysms and in areas with smaller aneurysms resolved..$^{39}$ Patients without aneurysms or stenosis do not appear to have subsequent complications, while long-term atherosclerotic risk evidence is mixed, and the follow-up needs to be done according to the clinical presentations..$^{20,30}$

\section{Conclusion}

Due to the orofacial manifestations, the participation of dentists, especially those specializing in oral medicine and pediatric dentistry, is essential for the early diagnosis of KD. In the face of the crisis caused by COVID-19 and the subsequent sequelae caused by the virus, it is necessary to consider the orofacial lesions related to KD. This is the first paper drawing attention to the importance of recognizing KD and KD-like symptoms in dentistry. Further studies will be needed to better understand the relationship between SARS-CoV-2 and these conditions.

\section{Acknowledgment}

The Minas Gerais State Research Foundation (FAPEMIG, Minas Gerais, Brazil), the National Council for Scientific and Technological Development (CNPq, Brazil) and the Coordination of Training of Higher Education Graduate Foundation (CAPES, Brazil).

\section{References}

1. Luca NJ, Yeung RS. Epidemiology and management of Kawasaki disease. Drugs. 2012 May;72(8):1029-38. https://doi.org/10.2165/11631440-000000000-00000

2. Makino N, Nakamura Y, Yashiro M, Kosami K, Matsubara Y, Ae R, et al. Nationwide epidemiologic survey of Kawasaki disease in Japan, 2015-2016. Pediatr Int (Roma). 2019 Apr;61(4):397-403. https://doi.org/10.1111/ped.13809

3. Dajani AS, Taubert KA, Gerber MA, Shulman ST, Ferrieri P, Freed M, et al. Diagnosis and therapy of Kawasaki disease in children. Circulation. 1993 May;87(5):1776-80. https://doi.org/10.1161/01.cir.87.5.1776

4. Singh S, Jindal AK, Pilania RK. Diagnosis of Kawasaki disease. Int J Rheum Dis. 2018 Jan;21(1):36-44. https://doi.org/10.1111/1756-185X.13224

5. Burns JC, Kushner HI, Bastian JF, Shike H, Shimizu C, Matsubara T, et al. Kawasaki disease:a brief history. Pediatrics. 2000 Aug;106(2):E27. https://doi.org/10.1542/peds.106.2.e27

6. Turnier JL, Anderson MS, Heizer HR, Jone PN, Glodé MP, Dominguez SR. Concurrent respiratory viruses and Kawasaki disease. Pediatrics. 2015 Sep;136(3):e609-14. https://doi.org/10.1542/peds.2015-0950

7. Jones VG, Mills M, Suarez D, Hogan CA, Yeh D, Bradley Segal J, et al. COVID-19 and Kawasaki disease: novel virus and novel case. Hosp Pediatr. 2020 Jun;10(6):537-40. https://doi.org/10.1542/hpeds.2020-0123

8. Cheung EW, Zachariah P, Gorelik M, Boneparth A, Kernie SG, Orange JS, et al. Multisystem inflammatory syndrome related to COVID-19 in previously healthy children and adolescents in New York City. JAMA. 2020 Jul;324(3):294-6. https://doi.org/10.1001/jama.2020.10374 
9. McCrindle BW, Manlhiot C. SARS-CoV-2-related inflammatory multisystem syndrome in children: different or shared etiology and pathophysiology as Kawasaki disease? JAMA. 2020 Jul;324(3):246-8. https://doi.org/10.1001/jama.2020.10370

10. Rivera-Figueroa El, Santos R, Simpson S, Garg P. Incomplete Kawasaki disease in a Child with Covid-19. Indian Pediatr. 2020 Jul;57(7):680-1. https://doi.org/10.1007/s13312-020-1900-0

11. Verdoni L, Mazza A, Gervasoni A, Martelli L, Ruggeri M, Ciuffreda M, et al. An outbreak of severe Kawasaki-like disease at the Italian epicentre of the SARS-CoV-2 epidemic: an observational cohort study. Lancet. 2020 Jun;395(10239):1771-8. https://doi.org/10.1016/S0140-6736(20)31103-X

12. Whittaker E, Bamford A, Kenny J, Kaforou M, Jones CE, Shah P, et al. Clinical characteristics of 58 children with a pediatric inflammatory multisystem syndrome temporally associated with SARS-CoV-2. JAMA. 2020 Jul;324(3):259-69. https://doi.org/10.1001/jama.2020.10369

13. The Lancet. COVID-19 in Brazil: "So what?". Lancet. 2020 May;395(10235):1461. https://doi.org/10.1016/S0140-6736(20)31095-3

14. Ayusawa M, Sonobe T, Uemura S, Ogawa S, Nakamura Y, Kiyosawa N, et al. Revision of diagnostic guidelines for Kawasaki disease (the 5th revised edition). Pediatr Int. 2005;47(2):232-4. https://doi.org/10.1111/j.1442-200x.2005.02033.x

15. McCrindle BW, Rowley AH, Newburger JW, Burns JC, Bolger AF, Gewitz M, et al. Diagnosis, treatment, and long-term management of Kawasaki disease: a scientific statement for health professionals from the American Heart Association. Circulation. 2017 Apr;135(17):e927-99. https://doi.org/10.1161/CIR.0000000000000484

16. Correction to: Diagnosis, treatment, and long-term management of Kawasaki disease: a scientific statement for health professionals from the American Heart Association. Circulation. 2019 Jul;140(5):e181-4. https://doi.org/10.1161/CIR.0000000000000703

17. Kawasaki T. [Acute febrile mucocutaneous syndrome with lymphoid involvement with specific desquamation of the fingers and toes in children]. Arerugi. 1967 Mar;16(3):178-222. Japanese.

18. Cohen E, Sundel R. Kawasaki disease at 50 years. JAMA Pediatr. 2016 Nov; 170(11):1093-9. https://doi.org/10.1001/jamapediatrics.2016.1446

19. Scuccimarri R. Kawasaki disease. Pediatr Clin North Am. 2012 Apr;59(2):425-45. https://doi.org/10.1016/i.pcl.2012.03.009

20. Newburger JW, Takahashi M, Gerber MA, Gewitz MH, Tani LY, Burns JC, et al. Diagnosis, treatment, and long-term management of Kawasaki disease: a statement for health professionals from the Committee on Rheumatic Fever, Endocarditis and Kawasaki Disease, Council on Cardiovascular Dlsease in the Young, America Heart Association. Circulation. 2004 Oct;1 10(17):2747-71. https://doi.org/10.1161/01.CIR.0000145143.19711.78

21. Cox JR, Sallis RE. Recognition of kawasaki disease. Perm J. 2009;13(1):57-61. https://doi.org/10.7812/TPP/08-042

22. Agarwal S, Agrawal DK. Kawasaki disease: etiopathogenesis and novel treatment strategies. Expert Rev Clin Immunol. 2017 Mar;13(3):247-58. https://doi.org/10.1080/1744666X.2017.1232165

23. Chang LY, Lu CY, Shao PL, Lee PI, Lin MT, Fan TY, et al. Viral infections associated with Kawasaki disease. J Formos Med Assoc. 2014 Mar;113(3):148-54. https://doi.org/10.1016/i.jfma.2013.12.008

24. Dong $Y, M_{0} X, H_{\cup} Y, Q_{i} X$, Jiang F, Jiang Z, et al. Epidemiological characteristics of 2143 pediatric patients with 2019 coronavirus disease in China. Pediatrics. 2020 Jun;145(6):e20200702. https://doi.org/10.1542/peds.2020-0702

25. Xu S, Chen M, Weng J. COVID-19 and Kawasaki disease in children. Pharmacol Res. 2020 Sep;159:104951. https://doi.org/10.1016/i.phrs.2020.104951

26. Toubiana J, Poirault C, Corsia A, Bajolle F, Fourgeaud J, Angoulvant F, et al. Kawasaki-like multisystem inflammatory syndrome in children during the covid-19 pandemic in Paris, France: prospective observational study. BMJ. 2020 Jun;369:m2094. https://doi.org/10.1136/bmi.m2094

27. Labé $P$, Ly A, Sin C, Nasser M, Chapelon-Fromont E, Ben Saïd P, et al. Erythema multiforme and Kawasaki disease associated with COVID-19 infection in children. J Eur Acad Dermatol Venereol. 2020 Oct;34(10):e539-41. https://doi.org/10.1111/idv.16666

28. Moreira A. Kawasaki disease linked to COVID-19 in children. Nat Rev Immunol. 2020 Jul;20(7):407. https://doi.org/10.1038/s41577-020-0350-1

29. Yoshino A, Tanaka R, Takano T, Oishi T. Afebrile Kawasaki disease with coronary artery dilatation. Pediatr Int (Roma). 2017 Mar;59(3):375-7. https://doi.org/10.1111/ped.13214

30. Saguil A, Fargo M, Grogan S. Diagnosis and management of kawasaki disease. Am Fam Physician. 2015 Mar;91(6):365-71.

31. Kushner HI, Macnee R, Burns JC. Impressions of Kawasaki syndrome in India. Indian Pediatr. 2006 Nov;43(11):939-42.

32. Chao SM, Phua KB. Perineal eruption as an early sign of Kawasaki disease. Ann Acad Med Singap. 1991 Mar;20(2):244-7.

33. Bhatnagar SK, Paul G, Subramanian R, Al Hosni MS, Al Khusaiby SM. Kawasaki disease in Oman: a clinical study. J Trop Pediatr. 2003 Dec;49(6):361-6. https://doi.org/10.1093/tropej/49.6.361

34. Scardina GA. Oral manifestation of Kawasaki disease. Res J Biol Sci. 2007;2(4):431-3.

35. Li X, Chen Y, Tang Y, Ding Y, Xu Q, Sun L, et al. Predictors of intravenous immunoglobulin-resistant Kawasaki disease in children: a meta-analysis of 4442 cases. Eur J Pediatr. 2018 Aug;177(8):1279-92. https://doi.org/10.1007/s00431-018-3182-2 
- Potential link between SARS-CoV-2 and Kawasaki disease: importance of dentists for the diagnosis

36. Verma L, Passi S, Kaur G, Gupta J, Joshi M. Recurrent Kawasaki disease presenting to dentists: "think beyond dentition". Int J Clin Pediatr Dent. 2018 Nov-Dec;11(6):532-5. https://doi.org/10.5005/ip-journals-10005-1571

37. Faden A. Recurrent lip swelling as a late presentation of Kawasaki disease: case report and review of literature. Saudi Dent J. 2013 Jan;25(1):43-7. https://doi.org/10.1016/i.sdentj.2012.10.001

38. Tremoulet AH, Best BM, Song S, Wang S, Corinaldesi E, Eichenfield JR, et al. Resistance to intravenous immunoglobulin in children with Kawasaki disease. J Pediatr. 2008 Jul;153(1):117-21. https://doi.org/10.1016/i.jpeds.2007.12.021

39. Gersony WM. The adult after kawasaki disease the risks for late coronary events. J Am Coll Cardiol. 2009 Nov;54(21):1921-3. https://doi.org/10.1016/j.jacc.2009.06.057 